\title{
Fumaric acid and succinic acid treat gestational hypertension by downregulating the expression of KCNMB1 and TET1
}

\author{
YIYUAN ZHOU, FANG ZHANG, HUIJIAO JIANG, DI XU and DONGYANG DENG \\ Department of Obstetrics, The First Affliated Hospital of Guizhou University of Traditional Chinese Medicine, \\ Guiyang, Guizhou 550001, P.R. China
}

Received January 10, 2019; Accepted November 11, 2019

DOI: $10.3892 /$ etm.2021.10506

\begin{abstract}
The present study hypothesized that fumaric acid and succinic acid may exhibit therapeutic effects on gestational hypertension. During pregnancy, estrogen upregulates ten-eleven translocation 1 (TET1) expression, which subsequently increases calcium-activated potassium channel subunit $\beta 1$ (KCNMB1) expression. KCNMB1 is associated with hypertension. Fumaric acid and succinic acid are understood to inhibit TET. Therefore, the present study investigated whether fumaric acid and succinic acid exhibit therapeutic effects on gestational hypertension and whether these effects are mediated by TET1 and KCNMB1. N $\omega$-Nitro-L-arginine methyl ester hydrochloride was injected into rats to establish a gestational hypertension model. Dimethyl fumarate (DMF) and succinic acid were administrated into rats to treat gestational hypertension. Rats were divided into five groups: i) Control; ii) model; iii) DMF; iv) succinic acid; and v) DMF + succinic acid. Blood pressure was monitored by a noninvasive meter and urinary protein was determined using a urinary protein kit. Placenta pathology was examined by hematoxylin-eosin staining. Compared with the control group, urinary protein and blood pressure in the model group increased significantly. The placental cells in the control group were arranged orderly. However, in the model group, decidual cellular edema of placenta and vacuolar degeneration were observed, and the intervascular membrane was markedly thicker with plenty of fibrin deposition. These results indicate successful establishment of a gestational hypertension model. However, compared with the model group, urinary protein, blood pressure, edema, vacuoles and fibrin deposition were markedly reduced in the DMF, succinic acid and DMF + succinic acid groups. mRNA
\end{abstract}

Correspondence to: Professor Dongyang Deng, Department of Obstetrics, The First Affliated Hospital of Guizhou University of Traditional Chinese Medicine, 71 Baoshanbei Road, Guiyang, Guizhou 550001, P.R. China

E-mail: ddydc1982@126.com

Key words: gestational hypertension, ten-eleven translocation 1, calcium-activated potassium channel subunit $\beta 1$, fumaric acid, succinic acid and protein levels of TET1 and KCNMB1 in placenta were evaluated by immunohistochemical analysis, reverse transcription-quantitative polymerase chain reaction and western blotting. The TET1 and KCNMB1 levels in the model group were markedly increased compared with those in the control group. However, compared with the model group, the expression levels were markedly downregulated in the DMF, succinic acid and DMF + succinic acid groups. In conclusion, fumaric acid and succinic acid may treat gestational hypertension by downregulating the expression of KCNMB1 and TET1.

\section{Introduction}

Gestational hypertension is a common obstetric disease, occurring in 5-10\% of pregnancies (1). Maternal death induced by gestational hypertension accounts for $10-16 \%$ of all pregnancy-associated mortalities and gestational hypertension is the second leading cause of maternal death in China (1). Hypertension, proteinuria and edema are the main symptoms of this condition. Gestational hypertension may be induced by maternal, placental and fetal factors, such as abnormal invasion of trophoblasts, abnormal immune regulation, endothelial cell damage, genetic factors and nutritional factors (2). No single factor can explain the complex etiology and mechanism of gestational hypertension (3). In the treatment of gestational hypertension, blood pressure must be lowered and severe preeclampsia and eclampsia must be prevented (4).

Metoprolol succinate is a selective $\beta 1$ receptor blocker. The dose required for its action on cardiac $\beta 1$ receptors is lower than that required for its action on peripheral blood vessels and bronchi $\beta 2$ receptors (5). Metoprolol succinate doesn't activate $\beta$ receptors on membranes. Metoprolol succinate can attenuate the effect of catecholamines associated with physiological and psychological loads, and reduce the heart rate, cardiac output and blood pressure (5). Bisoprolol fumarate is also a highly selective $\beta 1$ adrenoceptor antagonist, without intrinsic sympathomimetic and membrane stabilization activities. Bisoprolol fumarate has a high affinity for $\beta 1$ receptors of bronchi and vascular smooth muscle, which leads to vasodilation and lower blood pressure (5). Metoprolol succinate and bisoprolol fumarate are widely used in the treatment of hypertension (5). In addition, during pregnancy, estrogen upregulates ten-eleven translocation 1 (TET1) expression in uterine arteries, which activates demethylation and subsequently increases 
calcium-activated potassium channel subunit $\beta 1$ (KCNMB1) expression (6). KCNMB1 is associated with hypertension (7). Fumaric acid and succinic acid are known to inhibit TET (8). Therefore, the present study hypothesized that fumaric acid and succinic acid may exhibit therapeutic effects on gestational hypertension, and the present study was performed for preliminary confirmation.

The TET1 gene is a research hotspot at present for its special role in DNA methylation (9). TET1 is a hydroxymethylase, originally discovered as a fusion protein of histone H3K4 methyltransferase (10). TET1 can bind to the $\mathrm{CpG}$ region in the genome, which hinders catalytic activity of DNA methyltransferase and activates gene transcription (11). In addition, TET1 can recruit polycomb repressive complex 2 to the promoter region of a target gene, which ultimately suppressed the expression of the target gene (12). The TET protein family participates in the whole embryonic development process. When TET1 and TET3 are knocked-out at the same time, the transcriptome diversity increases during early embryonic development (13). Growth defects, increased mortality and developmental retardation are identified in the offspring of paternal mice following TET1-knockdown (14). BKCa channels are involved in the regulation of cellular functions, including neurotransmitter release, hormone secretion, heart rate, vascular stress resistance and smooth muscle tension, for their multiple regulatory characteristics. BKCa is composed of an ion-mediated $\alpha$ subunit and different $\beta$ subunits. The $\beta 1$ subunit is predominantly expressed in smooth muscle cells and it affects the behavior of these cells (15). Mutations in the KCNMBl gene encoding the $\beta 1$ subunit are associated with heart rate variability and baroreflex function (16). Variation of the KCNMBl gene loci decreases arterial impedance (17). $\mathrm{KCNMBl}$ may serve an important role in the development and progression of hypertension (18).

Therefore, the present study established a rat gestational hypertension model and investigated whether fumaric acid and succinic acid exhibit therapeutic effects on gestational hypertension and whether these effects are mediated by TET1 and KCNMBl.

\section{Materials and methods}

Materials and animals. N $\omega$-Nitro-L-arginine methyl ester hydrochloride (L-NAME; cat. no. 51298-65.5) and dimethyl fumarate (DMF; cat. no. C1821194) were purchased from Shanghai Aladdin Biochemical Technology Co., Ltd. Succinic acid (cat. no. ZN1113EA14) was obtained from Shanghai Yuanye Bio-Technology Co., Ltd. The rat urinary protein kit (cat. no. CO35-2) was obtained from Nanjing Jiancheng Bioengineering Institute. The bicinchoninic acid (BCA) kit (cat. no. CW0014), diaminobenzidine (DAB) kit (cat. no. CW0125), TRIzol reagent (cat. no. CW0580), Ultrapure RNA extraction kit (cat. no. CW0581), HiFiScript cDNA synthesis kit (cat. no. CW2569) and ULtraSYBR Mixture (cat. no. CW0957) were purchased from CoWin Biosciences Co., Ltd. (CWBIO). RIPA lysis buffer (cat. no. C1053) was purchased from Applygen Technologies, Inc. SuperSignal ${ }^{\circledR}$ west pico chemiluminescent substrate (cat. no. RJ239676) was obtained from Thermo Fisher Scientific, Inc. The polyvinylidene fluoride (PVDF) membrane (cat. no. IPVH00010) was obtained from EMD Millipore. Mouse anti-GAPDH monoclonal antibody (cat. no. TA-08; 1:2,000), horseradish peroxidase (HRP) conjugated goat anti-mouse $\operatorname{IgG}(\mathrm{H}+\mathrm{L})$ (cat. no. ZB-2305; 1:2,000) and HRP conjugated goat anti-rabbit IgG $(\mathrm{H}+\mathrm{L})$ (cat. no. ZB-2301; 1:2,000) were purchased from OriGene Technologies, Inc. Rabbit anti-TET1 polyclonal antibody (cat. no. DF6428; 1:500) and rabbit anti-KCNMB1 polyclonal antibody (cat. no. DF-9301; 1:1,000) were purchased from Affinity Biosciences. Rabbit anti-KCNMB1 polyclonal antibody (cat, no. bs-7689R; 1:1,000) was obtained from BIOSS.

In total, 35 female (three months-old, 250-280 g) and 20 male (three months-old, 400-500 g) Sprague-Dawley rats were obtained from the Hunan SJA Laboratory Animal Co., Ltd. [license no. SCXK(Xiang)2016-0002]. All rats were provided with free access to food and water and kept in conditions of $22-25^{\circ} \mathrm{C}$ under a 12 -h light/dark cycle. During the experimental phase, animal well-being was monitored by individual ventilated caging system (SmartRack, BioZone). Unpregnant rats and male rats after mating was completed and the fetuses after birth were fed normally and sacrificed at the end of the whole experiment. The experiments did not affect normal activity and eating behavior of rats. Rats were sacrificed after anesthesia. The study protocol was reviewed and approved by the Ethics Committee of the First Affiliated Hospital of Guiyang University of Chinese Medicine.

Gestational hypertension model establishment. Overnight, female and male rats were placed in a cage at a ratio of 2:1 to induce pregnancy. Formation of vaginal plug was observed the next morning. A total of 30 female rats were impregnated. Rats with vaginal plug were immediately placed in separate cages and day 0 of pregnancy was record. Blood pressure was measured on day 9 of pregnancy using a constant temperature noninvasive blood pressure meter (XH200; Beijing Zhongshidichuang Co., Ltd.). The rats were then randomly grouped $(n=6)$. Drugs were administrated by gavage at $10 \mathrm{ml} / \mathrm{kg}$ q.o.d, from day 10 of pregnancy to the birth of the fetus. Concentrations of DMF and succinic acid were 2.5 and $40 \mathrm{mg} / \mathrm{ml}$, respectively. Rats in the control and model groups were not treated with drugs. Subcutaneous injection of L-NAME into the back was performed continuously at $125 \mathrm{mg} / \mathrm{kg}$ q.o.d. for 5 days from day 14 of pregnancy to induce gestational hypertension. Blood pressure was measured again on day 19 of pregnancy. Finally, rats were anesthetized by intraperitoneal injection of $10 \%$ chloral hydrate at $350 \mathrm{mg} / \mathrm{kg}$. No signs of peritonitis were observed. Urine was collected by abdominal compression and punctio vesicae. After the fetus was born, rats were sacrificed by cervical dislocation and the placenta was collected. The urine and a portion of the placenta were stored at $-80^{\circ} \mathrm{C}$. Another portion of the placenta was fixed in $4 \%$ paraformaldehyde at room temperature for $24 \mathrm{~h}$ and stored at room temperature.

Experimental grouping. A total of 30 pregnant rats were divided into five groups (n=6): i) Control; ii) model; iii) DMF; iv) succinic acid; and v) DMF + succinic acid. Rats without any treatment served as the control group. Rats in the model group received L-NAME injection but did not receive any drug treatment. Rats that had gestational hypertension and 
were treated with DMF were assigned as the DMF group. Rats that had gestational hypertension and were treated with succinic acid were assigned as the succinic acid group. Rats that had gestational hypertension and were treated with both DMF and succinic acid were assigned as the DMF + succinic acid group. In the DMF + succinic acid group, the doses of the two drugs were halved.

Urinary protein determination. Urinary protein was determined using a urinary protein kit, according to the manufacturer's protocol. Briefly, $50 \mu \mathrm{l}$ water, protein standard solution $(563 \mathrm{mg} / \mathrm{l})$ and the urine sample were added into a blank well, standard well and test well, respectively. Coomassie brilliant blue solution ( $3 \mathrm{ml}$ ) was added into each well, followed by mixing. After $5 \mathrm{~min}$, the absorbance (optical density value) was measured at $595 \mathrm{~nm}$ using a microplate reader (RT-6100; Rayto Life and Analytical Sciences Co., Ltd.).

Hematoxylin and eosin $(H \& E)$ staining. Tissues were fixed in $4 \%$ paraformaldehyde at room temperature, washed with running water, dehydrated in 70,80 and 90\% ethanol solution, immersed in an equivalent mixture of absolute ethanol and xylene for $15 \mathrm{~min}$, and transparentized in xylene twice for 15 min each time until transparency. After immersing in an equivalent mixture of xylene and paraffin for $15 \mathrm{~min}$ and then in paraffin twice for 50-60 min each time, the tissues were embedded in paraffin and cut into slices, $4-\mu \mathrm{m}$ thick. Subsequently, the slices were baked, dewaxed, hydrated and then stained with hematoxylin solution for $3 \mathrm{~min}$. Sections were then differentiated in alcoholic hydrochloric acid for $15 \mathrm{sec}$, washed slightly, blued in bluing buffer for $15 \mathrm{sec}$, washed with running water, stained in eosin solution at room temperature for $3 \mathrm{~min}$. After staining sections were washed with running water, dehydrated, transparentized, mounted with neutral resin and examined under a light microscope (CKX41; Olympus Corporation).

Immunohistochemical analysis. Tissue slices were prepared as mentioned above and baked at $65^{\circ} \mathrm{C}$ for $2 \mathrm{~h}$, immersed in xylene twice for $10 \mathrm{~min}$ each, and successively incubated in 100, 100, 95 and 80\% ethanol and water for 5 min each time. Subsequently, the slices were incubated in citrate buffer and heated for $2 \mathrm{~min}$ in a high-pressure cooker. The slices were then cooled naturally, rinsed with PBS, incubated in fresh $3 \%$ hydrogen peroxide at room temperature for $10 \mathrm{~min}$ and washed with PBS. After the excess PBS was absorbed by absorbent papers, $5 \%$ bovine serum albumin (Beijing Solarbio Science \& Technology, Co., Ltd.) was added dropwise onto the slices, which were then incubated at $37^{\circ} \mathrm{C}$ for $30 \mathrm{~min}$. After excess blocking buffer was absorbed by absorbent papers, the slices were incubated with primary antibody buffer (rabbit anti-TET1 polyclonal antibody, rabbit anti-KCNMB1 polyclonal antibody) at $4^{\circ} \mathrm{C}$ overnight. Following washing with PBS three times, sections were incubated with the secondary antibody buffer (horseradish peroxidase-conjugated goat anti-rabbit $\mathrm{IgG}$ ) at $37^{\circ} \mathrm{C}$ for $30 \mathrm{~min}$. Then sections were rinsed with PBS, developed for 5-10 min using a DAB kit, rinsed with PBS, stained in hematoxylin solution at room temperature for $3 \mathrm{~min}$. Alcoholic hydrochloric acid was used to differentiate and after that sections were blued in bluing buffer, rinsed with
Table I. The sequences of primers.

\begin{tabular}{llc}
\hline Primers & \multicolumn{1}{c}{ Sequences (5'-3') } & $\begin{array}{c}\text { Product } \\
\text { length (bp) }\end{array}$ \\
\hline TET1 & $\begin{array}{l}\text { F: AAACGGAAGTCAAAACCCC } \\
\text { R: CCGAAGAGCCATTGTAAACC }\end{array}$ & 140 \\
KCNMB1 & F: AACATCAAGGACCAGGAAGAG & \multirow{2}{*}{129} \\
& R: TTGGTTTGATCCCGAGTG & \\
GAPDH & F: GCAAGTTCAACGGCACAG & 141 \\
& R: CGCCAGTAGACTCCACGAC & \\
\hline
\end{tabular}

TET1, ten-eleven translocation 1; KCNMB1, calcium-activated potassium channel subunit $\beta ; \mathrm{F}$, forward; $\mathrm{R}$, reverse.

water, dehydrated, transparentized, mounted and examined under a light microscope (CKX41; Olympus Corporation).

Reverse transcription-quantitative polymerase chain reaction $(R T-q P C R)$. Tissue RNA was extracted using TRIzol reagent and RT to cDNA was conducted at $42^{\circ} \mathrm{C}$ using the HiFiScript cDNA synthesis kit according to their manufacturer's protocol. Table I presents the primer sequences. The PCR reaction was composed of $1 \mu \mathrm{l}$ cDNA/DNA, $1 \mu \mathrm{l}$ forward primer, $1 \mu \mathrm{l}$ reverse primer, 12.5 $\mu \mathrm{l}$ ULtraSYBR Mixture and 9.5 $\mu \mathrm{l}$ RNase free $\mathrm{dH}_{2} \mathrm{O}$. Reaction parameters included pre-denaturation at $95^{\circ} \mathrm{C}$ for $10 \mathrm{~min}$, denaturation at $95^{\circ} \mathrm{C}$ for $10 \mathrm{sec}$, annealing at $58.5^{\circ} \mathrm{C}$ for $30 \mathrm{sec}$, elongation at $72^{\circ} \mathrm{C}$ for $30 \mathrm{sec}(40$ cycles). Analysis parameters of the dissociation curve included $15 \mathrm{sec}$ at $95^{\circ} \mathrm{C}, 1 \mathrm{~min}$ at $58.5^{\circ} \mathrm{C}, 15 \mathrm{sec}$ at $95^{\circ} \mathrm{C}, 15 \mathrm{sec}$ at $58.5^{\circ} \mathrm{C}$ and $15 \mathrm{sec}$ at $58.5^{\circ} \mathrm{C}$, and measured stepwise from $95^{\circ} \mathrm{C}$, every $0.5^{\circ} \mathrm{C}$. Products were examined using a RT-qPCR detection system (CFX Connect ${ }^{\mathrm{TM}}$; Bio-Rad Laboratories, Inc.). GAPDH served as an internal control. Relative levels of genes were calculated using a $2^{-\Delta \Delta \mathrm{Cq}}$ method (19).

Western blotting. Tissues were lysed in RIPA lysis buffer at $4^{\circ} \mathrm{C}$ for $30 \mathrm{~min}$ and then centrifuged at $9,000 \times \mathrm{g}$ and $4^{\circ} \mathrm{C}$ for $10 \mathrm{~min}$. The supernatant was collected carefully to obtain total protein. Protein concentration was determined using a BCA kit. Subsequently, the protein $(24 \mu \mathrm{g})$ was denatured and separated by $12 \%$ SDS-PAGE for $1-2 \mathrm{~h}$. The protein was transferred to a PVDF membrane by a wet method for 30-50 min, which was then blocked with $3 \%$ skimmed milk at room temperature for $1 \mathrm{~h}$ and incubated with primary antibody buffer at $4^{\circ} \mathrm{C}$ overnight. After washing, the membrane was incubated with secondary antibody buffer at room temperature for $1-2 \mathrm{~h}$, incubated with chemiluminescent substrate and examined on a gel imaging system (ChemiDoc ${ }^{\mathrm{TM}}$ XRS+; Bio-Rad Laboratories, Inc.). Gray values were analyzed using Quantity One software (v4.62; Bio-Rad Laboratories, Inc.). GAPDH served as an internal control.

Statistical analysis. Data are presented as the mean \pm standard deviation. Every experiment was repeated three times. Data were statistically analyzed using one-way analysis of variance followed by Tukey's post-hoc test with SPSS 19.0 software (IBM, Corp.). $\mathrm{P}<0.05$ was considered to indicate a statistically significant difference. 

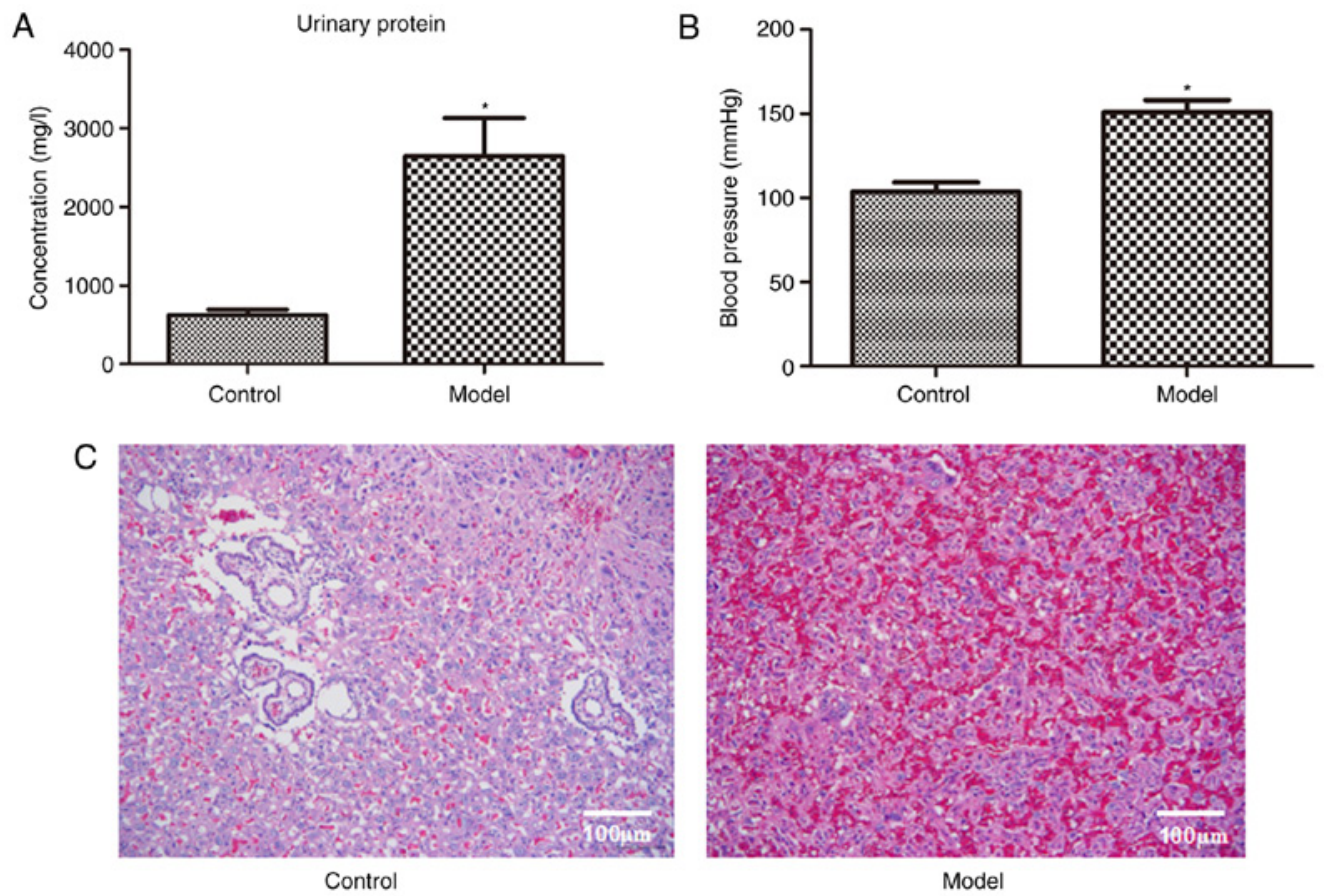

Figure 1. Characterization of gestational hypertension model. (A) Urinary protein, (B) blood pressure and (C) hematoxylin and eosin staining images of the placenta in the control and model rats. ${ }^{*} \mathrm{P}<0.05$ vs. the control.

\section{Results}

Establishment of a gestational hypertension model. Fig. 1 presents urinary protein (Fig. 1A), blood pressure (Fig. 1B) and H\&E staining images of the placenta (Fig. 1C) in the control and model rats. Compared with the control rats, urinary protein and blood pressure in the model rats increased significantly $(\mathrm{P}<0.05$; Fig. $1 \mathrm{~A}$ and $\mathrm{B})$. As presented in Fig. 1C, placental cells in the control rats were arranged in an orderly fashion. However, in the model rats, decidual cellular edema of the placenta and vacuolar degeneration were observed, and the intervascular membrane was obviously thicker with a large amount of fibrin deposition. These results indicate that the gestational hypertension model was successfully established.

Urinary protein and blood pressure. Fig. 2 presents the urinary protein (Fig. 2A) and blood pressure (Fig. 2B) of rats in various groups. Compared with the control group, urinary protein and blood pressure in the model group increased significantly $(\mathrm{P}<0.05)$. However, compared with the model group, urinary protein and blood pressure in the DMF, succinic acid and DMF + succinic acid groups decreased significantly $(\mathrm{P}<0.05)$.

$H \& E$ staining. Fig. 3 presents H\&E staining images of the placenta in the rats of various groups. Placental cells were arranged in an orderly manner in the control group. However, decidual cellular edema of the placenta, vacuolar degeneration, thicker intervascular membranes and a large amount of fibrin deposition were identified in the model group. Notably, compared with the model group, the edema, vacuoles and fibrin deposition were markedly reduced in the DMF, succinic acid and DMF + succinic acid groups.
Levels of TET1 and KCNMB1.mRNA and protein levels of TET1 and KCNMB1 in the placenta of various groups were examined by immunohistochemical analysis (Fig. 4A), RT-qPCR (Fig. 4B) and western blotting (Fig. 4C). The protein of interest is brown in the immunohistochemical images. Immunohistochemical analysis, RT-qPCR and western blot demonstrated similar results. The levels of TET1 and KCNMB1 were significantly increased in the model groups compared with the control group $(\mathrm{P}<0.05)$. However, compared with the model group, their levels were significantly downregulated in the DMF, succinic acid and $\mathrm{DMF}+$ succinic acid groups $(\mathrm{P}<0.05)$.

\section{Discussion}

At present, therapies for gestational hypertension are far from satisfactory. Establishment of an animal model provides a good basis for the investigation of treatments due to the relatively short gestation period in animals. However, the short gestation period and requirement of reserving enough time to treat the disease makes it difficult to establish a model. In the present study, a gestational hypertension model was established by subcutaneous injection of L-NAME. Results of urinary protein and blood pressure measurements demonstrated that urinary protein and blood pressure increased in the model rats. Furthermore, pathological examinations revealed the decidual cellular edema of the placenta, vacuolar degeneration, thicker intervascular membranes and a large amount of fibrin deposition in the model rats. These results confirmed the successful establishment of a gestational hypertension model. L-NAME is an inhibitor of nitric oxide (NO) synthase and can effectively inhibit NO synthesis. $\mathrm{NO}$ is an important active substance in maintaining homeostasis in humans and animals. A previous study reported that the NO level in pregnancy is higher than that in the normal state and inhibition of NO synthesis will lead to elevated blood pressure, 

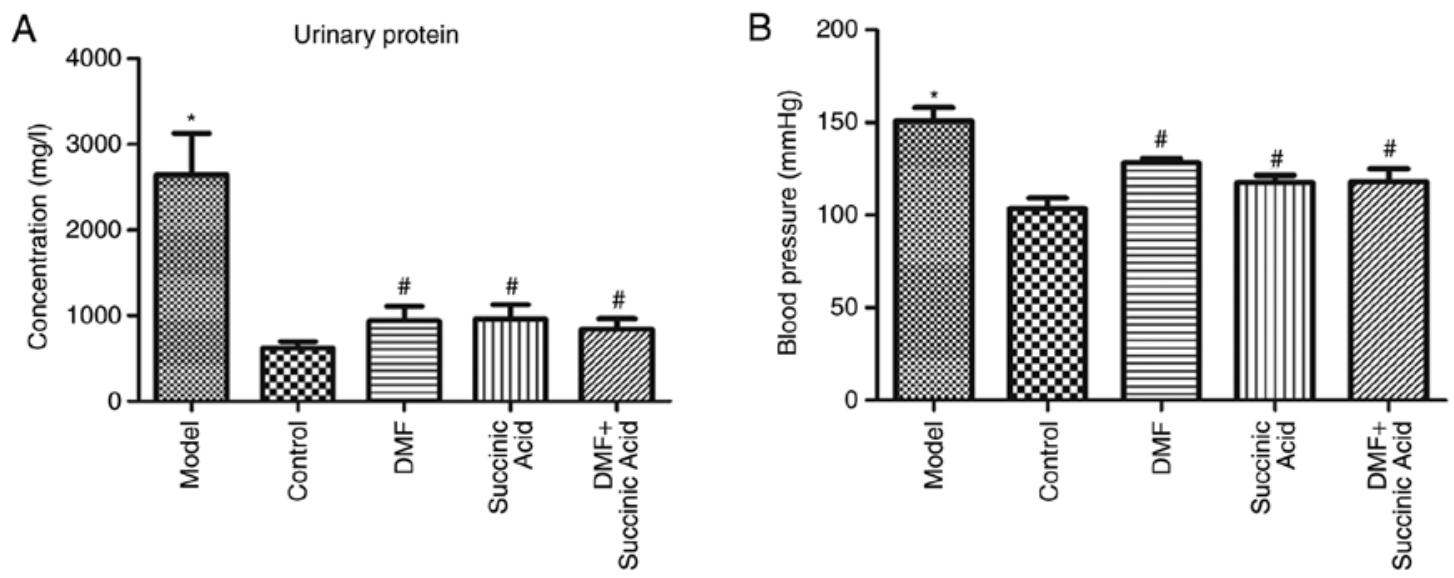

Figure 2. Urinary protein and blood pressure after drug treatment. (A) Urinary protein and (B) blood pressure of rats in the control, model, DMF, succinic acid and DMF + succinic acid groups. " $\mathrm{P}<0.05$ vs. the control; ${ }^{\#} \mathrm{P}<0.05$ vs. the model. $\mathrm{DMF}$, dimethyl fumarate.

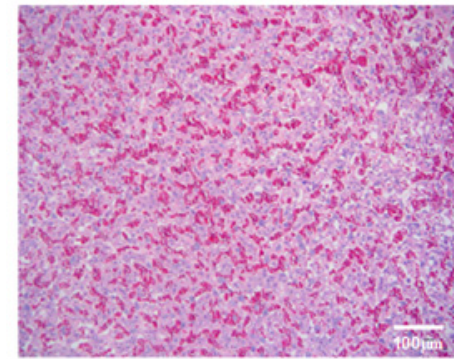

Model

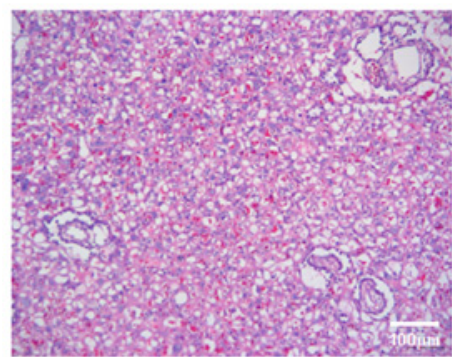

Succinic Acid

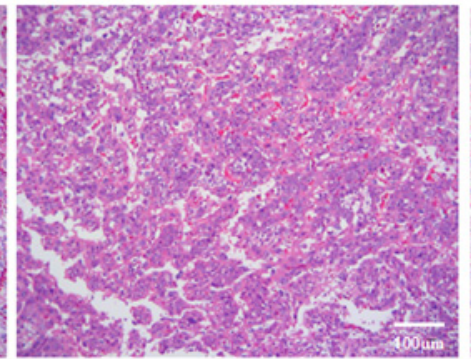

Control

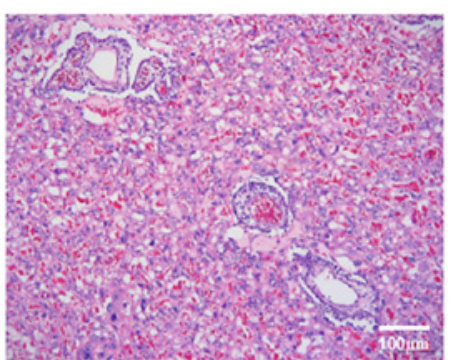

DMF+Succinic Acid

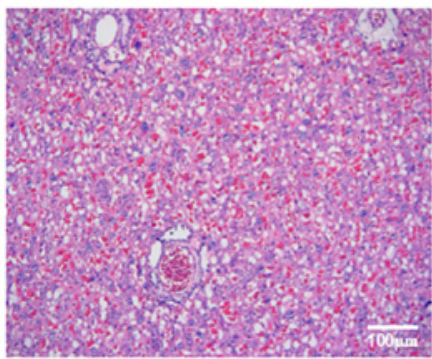

DMF

Figure 3. Hematoxylin and eosin staining images of placenta in the rats of the control, model, DMF, succinic acid and DMF + succinic acid groups. DMF, dimethyl fumarate.

proteinuria and symptoms of preeclampsia (20). The results of the present study agreed with this previous study. Elevated blood pressure may be a result of an enhanced response of the vascular system in pregnant rats to angiotensin $\mathrm{E}$ and norepinephrine by inhibiting NO synthesis (21).

The present study identified that urinary protein and blood pressure decreased, and placental cells in pregnant rats were improved during gestational hypertension when fumaric acid and succinic acid were administrated. These results suggest that fumaric acid and succinic acid exerted therapeutic effects on gestational hypertension. Non-toxic side effects of fumaric acid and succinic acid in rats are well recognized in previous reports $(22,23)$; therefore, the present study did not perform experiments to confirm their non-toxic side effects in normal non-pregnant female rats.

The TET protein family participates in the whole embryonic development process. When TET1 and TET3 are knocked-out at the same time, the transcriptome diversity increases during early embryonic development (13). Growth defects, increased mortality and developmental retardation are identified in the offspring of paternal mice following TET1-knockout (14). TET1 expression is upregulated during fetal growth, suggesting that TET1 gene may regulate early fetal growth (24). Numerous studies have reported that $\mathrm{KCNMB1}$ reduces the probability of a $\mathrm{BKCa}$ channel being open and instantaneous outward potassium current, which weakens the negative feedback inhibitory effect of the $\mathrm{BKCa}$ channel and consequently leads to depolarization of the cell membrane, contraction of vascular smooth muscle and elevation of blood pressure $(18,25)$. Correlation analysis between KCNMB1 mutation and hypertension demonstrated that KCNMB1 mutation results in increased sensitivity of the BK channel to calcium ions, which makes blood vessels easier to relax (26). Therefore, the KCNMB1 channel has a negative feedback effect on the contraction of vascular smooth muscle (26). During pregnancy, estrogen upregulates TET1 
A
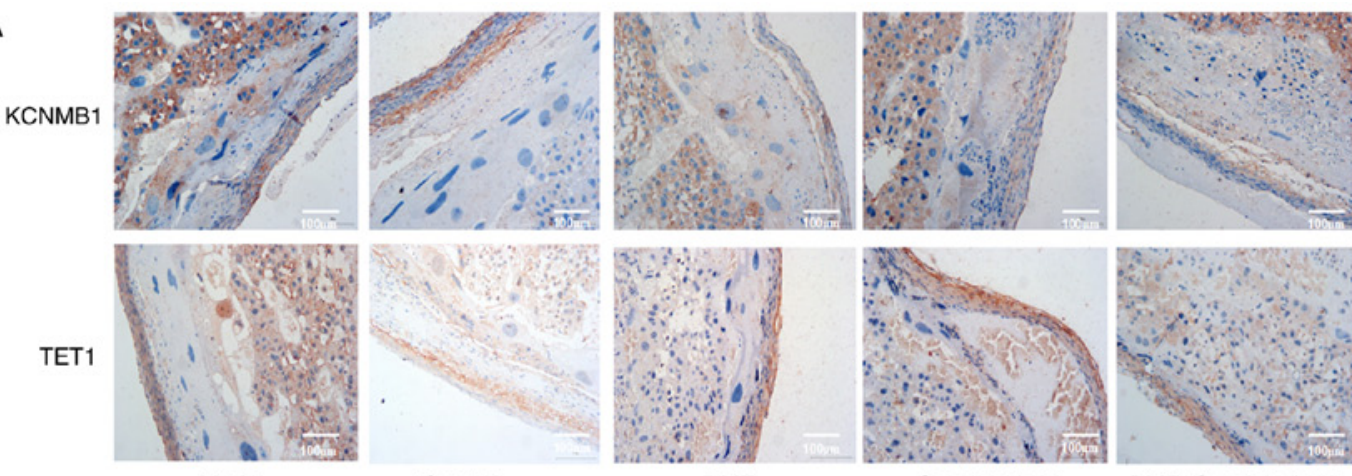

Model
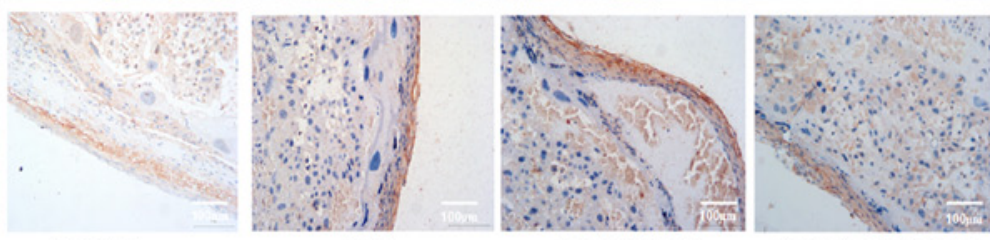

DMF

Succinic Acid

DMF+Succinic Acid
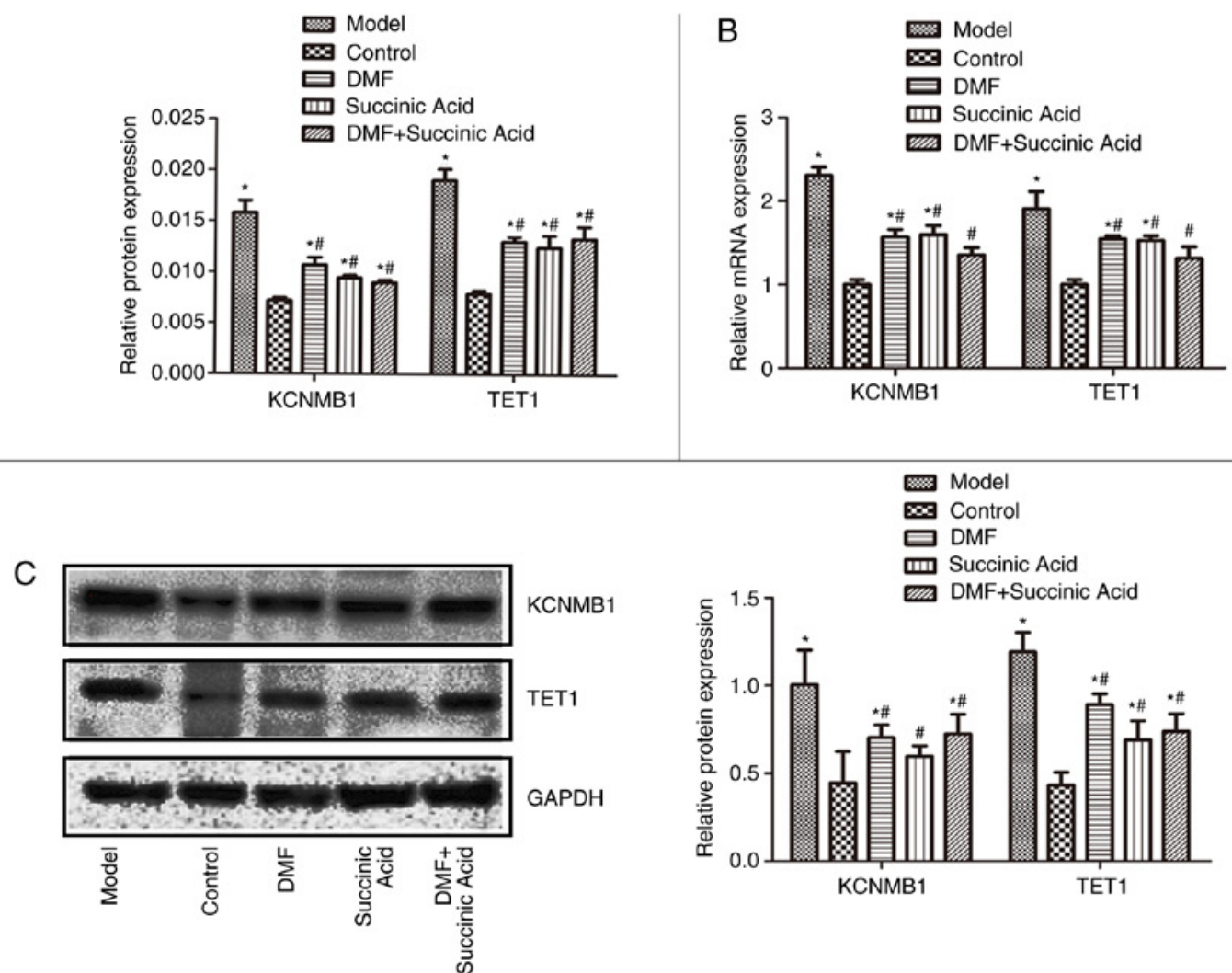

Figure 4. mRNA and protein levels of TET1 and KCNMB1 in the placenta of the control, model, DMF, succinic acid and DMF + succinic acid groups, which were examined by (A) immunohistochemical analysis, (B) reverse transcription-quantitative PCR and (C) western blotting. The protein of interest is brown in the immunohistochemical images. ${ }^{*} \mathrm{P}<0.05$ vs. control; ${ }^{\#} \mathrm{P}<0.05$ vs. model. DMF, dimethyl fumarate; TET1, ten-eleven translocation 1 ; KCNMB1, calcium-activated potassium channel subunit $\beta$.

expression in uterine arteries, which activates demethylation and subsequently increases KCNMB1 expression (6). KCNMB1 is associated with hypertension (7). The present results revealed that expression levels of TET1 and KCNMB1 increase during gestational hypertension. This is consistent with the aforementioned reports that TET1 expression is elevated during pregnancy and also suggests that KCNMB1 is associated with hypertension. Fumaric acid and succinic acid are known to inhibit TET (8). In the present study, the expression of TET1 and KCNMB1 decreased following the administration of fumaric acid and succinic acid, suggesting that fumaric acid and succinic acid downregulate the expression of TET1 and KCNMB1.

The absence of histological scoring data was a limitation of the current study. In future studies, promoters or inhibitors will be used to investigate the regulatory relationship between
KCNMB1 and TET1 in the treatment of gestational hypertension by fumaric acid and succinic acid, and detect some other preeclampsia-related marker genes/proteins. Additionally, in the present study there were no significant differences for all results between the three treatment groups. This may be due to dosage or other reasons. In the DMF + succinic acid group, the doses of the two drugs were halved, respectively. The reasons underlying the non-significant differences between the three treatment groups is uncertain and requires further experiments to clarify; therefore this will be an aim of future studies.

In conclusion, fumaric acid and succinic acid may treat gestational hypertension by downregulating the expression of KCNMB1 and TET1. Although this requires further confirmation by larger-scale experiments and clinical trials, these results may offer interesting and effective knowledge for the development of drugs to treat gestational hypertension. 


\section{Acknowledgements}

Not applicable.

\section{Funding}

The present study was supported by the Guizhou Provincial Science and Technology Foundation [grant no. QianKeHeLHZi(2016)7510].

\section{Availability of data and materials}

The datasets used and/or analyzed during the current study are available from the corresponding author on reasonable request.

\section{Authors' contributions}

YZ and DD designed the study, analyzed the data and wrote the paper. YZ, FZ, HJ, DX and DD performed the study and collected data. All authors read and approved the final manuscript.

\section{Ethics approval and consent to participate}

The study protocol was reviewed and approved by the Ethics Committee of the First Affiliated Hospital of Guiyang University of Chinese Medicine.

\section{Patient consent for publication}

Not applicable.

\section{Competing interests}

The authors declare that they have no competing interests.

\section{References}

1. Wang YA, Chughtai AA, Farquhar CM, Pollock W, Lui K and Sullivan EA: Increased incidence of gestational hypertension and preeclampsia after assisted reproductive technology treatment. Fertil Steril 105: 920-926.e2, 2016.

2. Egeland GM, Klungsøyr K, Øyen N, Tell GS, Næss $\varnothing$ and Skjærven R: Preconception cardiovascular risk factor differences between gestational hypertension and preeclampsia: Cohort Norway study. Hypertension 67: 1173-1180, 2016.

3. Hua X, Zhang J, Guo Y, Shen M, Gaudet L, Janoudi G, Walker M and Wen SW: Effect of folic acid supplementation during pregnancy on gestational hypertension/preeclampsia: A systematic review and meta-analysis. Hypertens Pregnancy 35: 447-460, 2016.

4. Hromadnikova I, Kotlabova K, Hympanova L and Krofta L: Gestational hypertension, preeclampsia and intrauterine growth restriction induce dysregulation of cardiovascular and cerebrovascular disease associated microRNAs in maternal whole peripheral blood. Thromb Res 137: 126-140, 2016.

5. Ripley TL and Saseen JJ: $\beta$-blockers: A review of their pharmacological and physiological diversity in hypertension. Ann Pharmacother 48: 723-733, 2014.

6. Hu XQ, Dasgupta C, Chen M, Xiao D, Huang X, Han L, Yang S, $\mathrm{Xu} \mathrm{Z}$ and Zhang L: Pregnancy reprograms large-conductance $\mathrm{Ca}^{2+}$-activated $\mathrm{K}^{+}$channel in uterine arteries: Roles of ten-eleven translocation methylcytosine dioxygenase 1-mediated active demethylation. Hypertension 69: 1181-1191, 2017.

7. Han YY, Wang LJ, Zhang L, Zhang WW, Ma KT, Li L and Si JQ: Association between potassium channel SNPs and essential hypertension in Xinjiang Kazak Chinese patients. Exp Ther Med 14: 1999-2006, 2017.
8. Laukka T, Mariani CJ, Ihantola T, Cao JZ, Hokkanen J, Kaelin WG Jr, Godley LA and Koivunen P: Fumarate and succinate regulate expression of hypoxia-inducible genes via TET enzymes. J Biol Chem 291: 4256-4265, 2016.

9. Wiehle L, Raddatz G, Musch T, Dawlaty MM, Jaenisch R, Lyko F and Breiling A: Tet1 and Tet2 protect DNA methylation canyons against hypermethylation. Mol Cell Biol 36: 452-461, 2015.

10. Choudhury SR, Cui Y, Lubecka K, Stefanska B and Irudayaraj J: CRISPR-dCas9 mediated TET1 targeting for selective DNA demethylation at BRCA1 promoter. Oncotarget 7: 46545-46556, 2016.

11. Thomson JP, Ottaviano R, Unterberger EB, Lempiäinen $H$, Muller A, Terranova R, Illingworth RS, Webb S, Kerr AR, Lyall MJ, et al: Loss of Tet1 associated 5-hydroxymethylcytosine is concomitant with aberrant promoter hypermethylation in liver cancer. Cancer Res 76: 3097-3108, 2016.

12. Somineni HK, Zhang X, Biagini Myers JM, Kovacic MB, Ulm A, Jurcak N, Ryan PH, Khurana Hershey GK and Ji H: Ten-eleven translocation 1 (TET1) methylation is associated with childhood asthma and traffic-related air pollution. J Allergy Clin Immunol 137: 797-805.e5, 2016.

13. Zhu X, GirardoD, Govek EE,John K, Mellén M,Tamayo P, Mesirov JP and Hatten ME: Role of Tet1/3 genes and chromatin remodeling genes in cerebellar circuit formation. Neuron 89: 100-112, 2016.

14. Pei YF, Tao R, Li JF, Su LP, Yu BQ, Wu XY, Yan M, Gu QL, Zhu ZG and Liu BY: TET1 inhibits gastric cancer growth and metastasis by PTEN demethylation and re-expression. Oncotarget 7: 31322-31335, 2016.

15. Du C, Zheng Z, Li D, Chen L, Li N, Yi X, Yang Y, Guo F, Liu W, $\mathrm{Xie} \mathrm{X}$, et al: $\mathrm{BKCa}$ promotes growth and metastasis of prostate cancer through facilitating the coupling between $\alpha v \beta 3$ integrin and FAK. Oncotarget 7: 40174-40188, 2016.

16. Friedman D, Kannan K, Faustin A, Shroff S, Thomas C, Heguy A, Serrano J, Snuderl M and Devinsky O: Cardiac arrhythmia and neuroexcitability gene variants in resected brain tissue from patients with sudden unexpected death in epilepsy (SUDEP). NPJ Genom Med 3: 9, 2018.

17. Díaz-Villamarín X, Dávila-Fajardo CL, Gónzalez-Medina MD, Soto-Pino MJ, Sánche-Gómez E, Acuña A, Gómez-Martín A, Martínez-González LJ, Casas-Hidalgo I and Cabeza-Barrera J: PKP-022 The KCNMB1 (A >G) (RS703505) genetic variant and the efficacy of tocilizumab in rheumatoid arthritis patients. Eur J Hosp Pharm Sci Pract 23 (Suppl 1): A188, 2016.

18. Hu XQ, Chen M, Dasgupta C, Xiao D, Huang X, Yang S and Zhang L: Chronic hypoxia upregulates DNA methyltransferase and represses large conductance $\mathrm{Ca}^{2+}$-activated $\mathrm{K}^{+}$channel function in ovine uterine arteries. Biol Reprod 96: 424-434, 2017.

19. Livak KJ and Schmittgen TD: Analysis of relative gene expression data using real-time quantitative PCR and the 2(- $\Delta \Delta \mathrm{C}(\mathrm{T}))$ method. Methods 25: 402-408, 2001.

20. Jin S, Teng X, Xiao L, Xue H, Guo Q, Duan X, Chen Y and Wu Y: Hydrogen sulfide ameliorated L-NAME-induced hypertensive heart disease by the Akt/eNOS/NO pathway. Exp Biol Med (Maywood) 242: 1831-1841, 2017.

21. Bogodvid TK, Andrianov VV,Muranova LN and Gainutdinov KL: Influence of nonspecific inhibitor of NO-synthase L-NAME on electric characteristics of premotor interneurons of terrestrial snails. Bionanoscience 8: 884-887, 2018.

22. Godbole SS, Kaul R, D'Souza SF and Nadkarni GB: Immobilization of fumarase by entrapment of rat liver mitochondria in polyacrylamide gel using gamma rays. Biotechnol Bioeng 25: 217-224, 1983.

23. Kirchgessner M, Roth FX, Tschierschwitz A and Grassmann E: Nutritive effect of fumaric acid on growth and body composition of rats. Z Tierphysiol Tierernähr Futtermittelkd 47: 175-186, 1982 (In German).

24. Kang J, Lienhard M, Pastor WA, Chawla A, Novotny M, Tsagaratou A, Lasken RS, Thompson EC, Surani MA, Koralov SB, et al: Simultaneous deletion of the methylcytosine oxidases Tet 1 and Tet 3 increases transcriptome variability in early embryogenesis. Proc Natl Acad Sci USA 112: E4236-E4245, 2015.

25. Yang GX, Fu HJ, Liu TW, Li JL, Shen Y and Qian J: Effects of atorvastatin on VSMC apoptosis in rabbits with atherosclerosis and its molecular mechanism. Chin J Arterioscler 25: 463-466, 2017.

26. Wang LJ, Zhang WW, Qian YF, Zhang L, Zhao L, Ma KT, Li L and Si JQ: Association between KCNMB1 polymorphism and essential hypertension in Xinjiang Kazak population. J Xian Jiaotong Univ 37: 78-81, 2016.

This work is licensed under a Creative Commons Attribution-NonCommercial-NoDerivatives 4.0 International (CC BY-NC-ND 4.0) License. 